\title{
Síndrome das Pernas Inquietas/Doença de Willis-Ekbom e infecção pelo HIV: revisão da epidemiologia e fisiopatologia
}

\author{
Restless Leg Syndrome/Willis-Ekbom Disease and HIV: a \\ review of the epidemiology and pathophysiology
}

Fernanda Gabrielle Almeida Castro ${ }^{1}$, Gilmar Fernandes do Prado ${ }^{2}$

1.Neurologista, Residente em Medicina do Sono, Setor Neuro-Sono, Disciplina de Neurologia, Escola Paulista de Medicina da Universidade Federal de São Paulo, São Paulo-SP, Brasil.

2.Neurologista, Professor Livre Docente, Setor Neuro-Sono, Disciplina de Neurologia, Escola Paulista de Medicina da Universidade Federal de São Paulo, São Paulo-SP, Brasil.

\begin{abstract}
Resumo
Introdução. A Síndrome das Pernas Inquietas/Doença de Willis Ekbom (SPI/DWE) é uma desordem neurológica comum, que pode perturbar profundamente o sono e a qualidade de vida. A prevalência da SPI/DWE varia entre 5-15\%. Sua fisiopatologia envolve a regulação da dopamina e ferro e, possivelmente, mecanismos inflamatórios. A prevalência de SPI parece estar aumentada em pacientes com infecção pelo HIV (Human Immunodeficiency Virus), e uma vez que a doença causa impacto significativo na qualidade do sono dos pacientes, faz-se necessário estudos maiores envolvendo esta associação. Objetivo. Revisar a literatura quanto a associação SPI/HIV. Método. Foi realizado uma revisão bibliográfica, através das bases de dados eletrônicas PUBMED, SCIELO e PERIÓDICO CAPES usando as palavras chaves: "HIV", "Restless leg Syndrome" e "Sleep disturbance". Resultados. Foram encontradas uma vasta quantidade de artigos, entretanto somente cinco, envolviam especificamente a associação. Foram usados um total de 25 artigos para desenvolvimento desta revisão. Conclusão. A prevalência da SPI/HIV varia entre $8 \%$ e $33 \%$. Dentre os mecanismos associados, podemos citar o envolvimento imunológico do SNC, presença de citocinas e polimorfismos e redução dos níveis de dopamina. A investigação de SPI/DWE nestes pacientes deve ser feita sempre que o paciente queixar-se de dificuldades de sono.

Unitermos. Síndrome das pernas inquietas; HIV; distúrbios do sono
\end{abstract}

\begin{abstract}
Introduction. Restless Legs Syndrome/Willis Ekbom Disease (RLS/WED) is a common neurological disorder that can profoundly disrupt sleep and quality of life. The prevalence of RLS is between $5-15 \%$. The pathophysiology involves dopamine and iron regulating and possibly inflammatory mechanisms. The prevalence of RLS appear to be increased in patients with HIV (Human Immunodeficiency Virus), and once the disease causes significant impact on patients' quality of sleep, it is necessary to further studies involving this association. Objective. To review the literature regarding the association RLS/HIV. Method. We conducted a literature review, through electronic databases PubMed, SCIELO and PERIODIC CAPES using the key words: "HIV", "Restless leg syndrome" and "Sleep disturbance." Results. A vast amount of articles were found, however only five specifically studied the association. We used a total of 25 review articles for its development. Conclusion. The prevalence of RLS/HIV varies between $8 \%$ and $33 \%$. Among the associated mechanisms, we can mention the immune CNS involvement, presence of cytokines and polymorphisms and reduced dopamine levels. The RLS/WED research in these patients should be performed whenever the patient complain of sleep difficulties.
\end{abstract}

Keywords. Restless Legs Syndrome; HIV; Sleep Disorders 
Trabalho realizado no Setor Neuro-Sono, Disciplina de Neurologia, Escola Paulista de Medicina da Universidade Federal de São Paulo, São Paulo-SP, Brasil.

\section{INTRODUÇÃO}

A Síndrome das Pernas Inquietas (SPI), também conhecida como Doença de Willis-Ekbom (DWE), é uma desordem neurológica sensitivo-motora comum, que pode perturbar profundamente o sono e a qualidade de vida. Como critérios diagnósticos é necessário que o paciente queixe-se de urgência para mover as pernas, usualmente acompanhada de sensação desconfortável nos membros, que iniciam ou pioram em períodos de inatividade, são aliviadas parcial ou totalmente pelo movimento e pioram durante a noite ${ }^{1}$. Estes sintomas podem causa estresse e significativo prejuízo social, ocupacional, educacional e em outras áreas, através do seu impacto sobre o sono, comportamento, cognição e humor ${ }^{1,2}$.

A prevalência da SPI/DWE varia entre 5-15\%, dependendo da população pesquisada ${ }^{3-6}$. Um estudo realizado na população brasileira encontrou uma prevalência de $6,4 \%^{6}$. Apresenta uma expressão clínica variada, influenciada por fatores genéticos, ambientais e médicos ${ }^{1}$. A síndrome pode ser classificada como primária ou secundária. SPI/DWE primária não tem causa aparente, exceto predisposição genética. Causas secundárias são resultado de certas condições clínicas, as mais descritas são 
deficiência de ferro, gravidez, insuficiência renal, diabetes, gravidez, neuropatias periféricas e doenças inflamatórias ${ }^{7-}$ 11.

A fisiopatologia da SPI/DWE ainda é pouco entendida. Estudos prévios tem demonstrado que sua fisiopatologia envolve a regulação da dopamina e ferro, com pacientes respondendo positivamente ao uso de medicações dopaminérgicas ${ }^{5,12,13}$. O ferro está integrado ao metabolismo da dopamina, e sua deficiência está relacionada com a etiologia da SPI/DWE.

A infecção pelo vírus da imunodeficiência humana (HIV) vem aumentando sua prevalência em todo mundo ${ }^{14}$. Globalmente, estima-se que 35,3 milhões de pessoas estão vivendo com o HIV. Embora a incidência esteja reduzindo, a prevalência em relação a anos anteriores aumentou devido maior uso de tratamento antirretroviral e maior sobrevida desses pacientes ${ }^{14}$. Aumenta-se desta forma as comorbidades e complicações decorrentes da infecção pelo vírus, dentre as outras, os distúrbios do sono.

Os distúrbios do sono são frequentemente relatados por pacientes infectados por HIV, com prevalências variáveis descritas na literatura. Estes indivíduos apresentam queixas frequentes de dificuldade de iniciar e manter o sono, sonolência excessiva diurna, fadiga e pior qualidade de sono ${ }^{15,16}$. Dentre os distúrbios do sono, podemos destacar a SPI/DWE, cuja prevalência nesta população varia de 8 a 33,3\%. Vários mecanismos parecem contribuir para o aumento da incidência de SPI/DWE nesta 
população, porém a literatura envolvendo esta associação é extremamente escassa ${ }^{17-20}$.

\section{MÉTODO}

Foi realizada uma revisão bibliográfica, usando as bases de dados eletrônicas MEDLINE, BIREME, SCIELO e PERIÓDICO CAPES. Utilizamos as palavras chaves: "HIV", "Restless Leg Syndrome" e "Sleep Disturbance". Foram incluídos artigos que abordavam a prevalência, fisiopatologia e apresentação clínica da SPI/DWE de maneira geral e da associação SPI/DWE com a infecção pelo HIV. Foram encontrados artigos em outras línguas, entretanto foram incluídos somente artigos publicados em português e inglês. Foram excluídos artigos, que abordavam somente 0 impacto dos transtornos psiquiátricos e medicações antirretrovirais no sono dos pacientes HIV positivos, artigos que abordavam somente tratamento da SPI, e aqueles referentes a outras complicações da infeç̧ão pelo HIV.

\section{RESULTADOS}

Usando as palavras chaves "HIV" e/ou "Restless Leg Syndrome" e/ou Sleep Disturbance", foram encontradas uma vasta quantidade de artigos, a maioria, entretanto, se referindo à associação HIV e outras doenças clínicas, efeito das medicações antirretrovirais, qualidade de sono, insônia, 
e influência de fatores como a depressão e dor sobre o sono. Realizando uma busca mais avançada, a partir de um refinamento sendo pesquisado as palavras chaves "HIV" e/and "Restless Leg Syndrome" foram encontrados apenas 13 artigos na literatura, desses, somente cinco artigos tratavam realmente desta associação, e destes somente três abordavam dados de prevalência (Tabela 1). Foram utilizados um total de 24 artigos para o desenvolvimento dessa revisão e dados epidemiológicos da AIDS, publicados no relatório global da AIDS (UNAIDS).

Os artigos incluídos foram publicados entre 2002 e 2014.

Tabela 1. Artigos abordando a prevalência da associação HIV e SPI/DWE.

\begin{tabular}{lcccc}
\hline Autor & Data & Local & N & Prevalência \\
\hline Happe S & & & & \\
\hline Hennessy & & & & \\
& 2007 & Alemanha & 129 & \\
\hline Lee $^{16}$ & 2014 & Califórnia & 316 & $11 \%$ \\
\hline
\end{tabular}

\section{DISCUSSÃO}

Os distúrbios do sono são frequentemente relatados por pacientes infectados por HIV. Um estudo publicado em 2014, com uma população de 1.354 pacientes em seis centros na Franca, encontrou uma prevalência de cerca de $47 \%$ nesta população ${ }^{15}$. Embora não tão vastas, existem 
publicações na literatura, referindo-se ao impacto da infecção por HIV na qualidade do sono, e aumento do risco de insônia e fadiga. Lee et al, em 2012, descreveram que $34 \%$ dos pacientes infectados da amostra, apresentam dificuldade para iniciar o sono, 56\% apresentam dificuldade para manter o sono e $20 \%$ apresentam ambos os problemas ${ }^{16}$. Quanto às causas desse quadro de insônia, a maioria dos estudos focam na questão de dor, medicações, transtornos de humor e conflitos sociais ${ }^{20-22}$. Entretanto, pouco tem sido descrito especificamente sobre a associação HIV e SPI/DWE.

Até o presente momento, foram encontrados 3 estudos, que dentre outros objetivos, avaliaram a prevalência de SPI/DWE em infectados por HIV. O primeiro estudo foi publicado em 2006 e realizado na Alemanha ${ }^{18}$. Foram incluídas 129 pessoas, destes 100\% eram caucasianos. Para diagnóstico de SPI/DWE os pacientes inicialmente responderam a um questionário com os critérios da doença e posteriormente foram avaliados por neurologistas experientes. Foram registradas as características epidemiológicas da população e os dados relacionados à infecção (contagem de linfócitos CD4, carga viral, estágio da doença e uso de terapia antirretroviral). Foi encontrada uma prevalência de 33,3\% de SPI/DWE nesta população, índices bem maiores ao descrito na literatura geral. A maioria dos pacientes eram homens, o que difere do observado na população geral (maior incidência no sexo feminino), porém reflete o fato de um 
número maior de portadores de HIV serem do sexo masculino. A escala de gravidade e a duração dos sintomas de SPI/DWE foram maiores em portadores de HIV em comparação aos controles e inversamente correlacionados com a contagem de linfócitos CD4 e com o índice de massa corpórea. Não houve correlação entre severidade da SPI/DWE e contagem viral, presença de polineuropatia, de infecções oportunistas, complexo demência-AIDS e uso de terapia antirretroviral. Foi encontrada uma associação de SPI/DWE e uma pior qualidade de sono e fadiga diurna. Os autores consideram que as alterações imunológicas e o tropismo do vírus pelo SNC possam predispor ao risco aumentado de SPI/DWE nesta população ${ }^{18}$. Embora neste estudo, não tenha sido encontrado uma maior prevalência de SPI/DWE em pacientes com polineuropatia periférica, os autores consideram ser possível que ela seja um dos fatores predisponentes para maior risco de SPI/DWE nesta população. Não há na literatura, entretanto, outros estudos que avaliam a relação especifica entre polineuropatia periférica e aumento da incidência de SPI/DWE nesta população.

Um estudo realizado com 316 pacientes infectados por HIV em São Francisco, Califórnia. A amostra era composta de uma maior diversidade étnica (caucasianos, negros e outros $)^{19}$. Os pacientes responderam a um questionário contendo os quatro critérios diagnósticos de SPI/DWE. Foi encontrada uma prevalência de $11 \%$ de SPI/DWE nesta população, o que é equiparável ao descrito na população 
geral. Foram registrados dados demográficos, clínicos, e laboratoriais (contagem de CD4, carga viral, níveis de hemoglobina e níveis de citocinas). A maioria eram homens, o que também refletiu o fato da maioria dos portadores de HIV serem homens nesta população. As únicas características que diferenciaram do grupo controle foram o recebimento de terapia com estrogênio e níveis inferiores de hemoglobina. Entretanto não foi objetivo determinar se os níveis menores de hemoglobina estavam associados a níveis reduzidos de ferro e ferritina suficientes para afetar o funcionamento do ferro no SNC. A contagem de linfócitos CD4 embora estivesse algo reduzido no grupo com SPI/DWE, não foram diferentes do grupo controle ${ }^{19}$.

A diferença de prevalência entre esses dois estudos, pode ter sido devido às diferenças raciais entre os dois estudos e às diferenças entre os métodos de diagnóstico de SPI/DWE.

Foram avaliados os tipos de distúrbios do sono que estavam presentes em pacientes com infecção pelo $\mathrm{HIV}^{16}$. O estudo encontrou uma prevalência de $8 \%$ de SPI/DWE, também inferior ao descrito no estudo alemão. Entretanto, não foi relatado o método usado para diagnóstico de SPI/DWE nesta população e não pareceu ser este um dos principais objetivos. Este estudo evidenciou ainda que pacientes com dificuldade para iniciar e manter o sono apresentavam menor contagem de linfócitos CD4 e maior carga viral que os controles ${ }^{16}$. 
Em relação aos mecanismos fisiopatológicos envolvidos na associação infecção pelo HIV e SPI/DWE, algumas hipóteses podem ser levantadas com base em algumas evidências já publicadas na literatura, envolvendo mecanismos imunes, atividade inflamatória e dopamina.

Algumas publicações sugerem que os distúrbios do sono são associados a baixos níveis de dopamina, o que pode levar ao aumento da sonolência e letargia. Um artigo publicado em $2013^{23}$ avaliou a concentração da dopamina urinária em pacientes infectados por HIV e a presença de distúrbios do sono. Através de aplicação de questionários sobre qualidade de sono, dosagem de carga viral e linfócitos T CD4 e quantificação da dopamina urinária de 24h, foi evidenciado que os distúrbios do sono nesta população, estão associados com uma contagem mais baixa de linfócitos CD4 (como já evidenciado em estudos anteriores) e níveis mais baixos de dopamina comparado com controles. Comprovando que a relação dopamina, sono e funcionamento imune são especialmente salientes no contexto HIV/AIDS ${ }^{23}$. Esta deficiência de dopamina pode estar envolvida também na predisposição dos pacientes HIV de desenvolverem sintomas de SPI.

Recentemente alguns estudos têm demonstrado a associação entre inflamação e distúrbios de manutenção do sono ${ }^{19,24,25}$. Doenças crônicas frequentemente estão associadas com processos inflamatórios e aumento de citocinas, particularmente o fator de necrose tumoral (TNFalfa) e interleucina 6 (IL-6). A infecção pelo HIV 
desencadeia alterações inflamatórias durante todo o curso da infecção. Durante a fase aguda, ocorre uma resposta inflamatória significativa representada pelo aparecimento de diversos marcadores plasmáticos de fase aguda e liberação de um grande número de citocinas inflamatórias.

Lee et al, em 2014 ${ }^{25}$, pesquisou citocinas e polimorfismos de genes de citocinas plasmáticas em 289 pacientes portadores de HIV/AIDS, os quais também tiveram seu padrão de sono monitorado por $72 \mathrm{~h}$ através de actigrafia. $O$ estudo concluiu que pacientes com polimorfismos genético das citocinas IL1R2 e TNF- alfa apresentam um maior tempo acordado após início do sono e redução do tempo total de sono, confirmando, portanto, a associação das citocinas com os distúrbios de sono em pacientes com HIV ${ }^{25}$.

As citocinas, que possuem a maior influência dentro dos processos inflamatórios, podem induzir sintomas associados com SPI-DWE por desregular os receptores de dopamina ${ }^{24}$.

Outro estudo recente, publicado em $2014^{19}$, teve como objetivo principal identificar biomarcadores de SPI-DWE em paciente com infecção pelo HIV. Em um total de 316 pacientes, foram coletadas amostras de sangue para análise plasmática dos níveis de citocinas TNF-alfa e proteína $C$ reativa e realizado genotipagem. O estudo evidenciou uma maior probabilidade de desenvolvimento de SPI em indivíduos que apresentavam duas cópias do alelo curto do gene IL1B (rs1143643), ou pelo menos uma cópia 
do alelo curto para IL17A (rs8193036). A interleucina IL17A regula a quantidade de ferro na hemoglobina. $A$ interleucina IL1B regula o efluxo de ferro das células gliais. Confirmando mais uma vez, a associação de citocinas e polimorfismos de citocinas na fisiopatologia de SPI/DWE em HIV positivos ${ }^{19}$.

\section{CONCLUSÃO}

A prevalência de SPI parece estar aumentada em indivíduos infectados por HIV. Dentre os mecanismos envolvidos, podemos citar o envolvimento imunológico do SNC, a presença de citocinas inflamatórias e polimorfismos de citocinas e a redução dos níveis de dopamina. A investigação de SPI nestes pacientes deve ser feita sempre que o paciente queixar-se de dificuldades de sono. Mais estudos devem ser realizados, com o objetivo de melhor compreensão da fisiopatologia envolvida.

\section{REFERÊNCIAS}

1.Allen RP, Picchietti DL, Garcia-Borreguero D, Ondo WG, Walters AS, Winkelman JW, et al. Restless legs syndrome/Willis-Ekbom disease diagnostic criteria: updated International Restless Legs Syndrome Study Group (IRLSSG) consensus criteria-history, rationale, description, and significance. Sleep Med 2014;15:860-73. http://dx.doi.org/10.1016/j.sleep.2014.03.025

2.Masuko AH, Carvalho LB, Machado MA, Morais JF Prado LB, Prado GF. Tradução e Validação para a Língua Portuguesa do Brasil da Escala Internacional de Graduação da Síndrome das Pernas Inquietas do Grupo Internacional de Estudos da Síndrome das Pernas Inquietas. Arq Neuropsiquiatr 2008;66:832-6. http://dx.doi.org/10.1590/s0004-282×2008000600011 
3.Tison F, Crochard A, Leger D, Bouee S, Lainey E, El Hasnaoui A. Epidemiology of restless legs syndrome in French adults a nationwide survey: the INSTANT Study. Neurology 2005;65:23946. http://dx.doi.org/10.1212/01.wnl.0000168910.48309.4a

4.Allen RP, Walters AS, Montplaisir J, Hening W, Myers A, Bell TJ, et al. Restless legs syndrome prevalence and impact: REST general population study. Arch Internal Med 2005;165:1286-92. http://dx.doi.org/10.1001/archinte.165.11.1286

5.Zucconi M, Ferini-Strambi L. Epidemiology and clinical findings of restless legs syndrome. Sleep Med 2004;5:293-9. http://dx.doi.org/10.1016/j.sleep.2004.01.004

6. Eckeli AL, Gitaí LL, Dach F, Ceretta H, Sander HH, Passos AD, et al. Prevalence of restless legs syndrome in the rural town of Cassia dos Coqueiros in Brazil. Sleep Med 2011;268:762-7. http://dx.doi.org/10.1016/j.sleep.2011.01.018

7.Allen RP. Controversies and challenges in defining the etiology and pathophysiology of restless legs syndrome. Am J Med 2007;120:S1321. http://dx.doi.org/10.1016/j.amjmed.2006.11.003

8.Galdino DA, Carvalho LB, Prado GF. Restless legs syndrome during pregnancy in Brazilian women. Sleep Med 2010;11:1049-54. http://dx.doi.org/10.1016/j.sleep.2010.06.006

9. Marin LF, Carvalho LB, Prado LB, Quadros AJ, Oliveira AS, Prado GF. Restless legs syndrome in post-polio syndrome: A series of 10 patients with demographic, clinical and laboratorial findings. Parkinsonism Rel Disor 2011;17:563-4. http://dx.doi.org/10.1016/j.parkreldis.2011.02.011

10.Weinstock LB, Walters AS, Paueksakon P. Restless legs syndrome: Theoretical roles of inflammatory and immune mechanisms. Sleep Med Rev 2012;16:341-54.

http://dx.doi.org/10.1016/j.smrv.2011.09.003

11.Prado GF, Allen RP, Trevisani VM, Toscano VG, Earley CJ. Sleep disruption in systemic sclerosis (scleroderma) patients: clinical and polysomnographic findings. Sleep Med 2002;3:341-5. http://dx.doi.org/10.1016/s1389-9457(02)00013-8

12. Hornyak M, Trenkwalder C, Kohnen R, Scholz H. Efficacy and safety of dopamine agonists in restless legs syndrome. Sleep Med 2012;13:228-36. http://dx.doi.org/10.1016/j.sleep.2011.09.013

13.Conti CF, De Oliveira MM, Andriolo RB, Saconato H, Atallah AN, Valbuza JS, et al. Levodopa for idiopathic restless legs syndrome: Evidence-based review. Mov Disord 2007;22:1943-51. http://dx.doi.org/10.1002/mds.21662

14.UNAIDS report on the global AIDS epidemic 2013 (endereço na internet). Switzerland: Unaids. Acessado em 2017. Disponível em: https://www.unaids.org/en/resources/documents/2013/20130923 U NAIDS Global Report 2013

15.Allavena C, Guimard T, Billaud E, De La Tullaye S, Reliquet V, Pineau $S$, et al. Prevalence and risk factors of sleep disturbances in a large HIV-infected adult population. J Int AIDS Soc $2014 ; 17$ (4 suppl 3): 19576. http://dx.doi.org/10.7448/IAS.17.4.19576 
16. Lee KA, Gay C, Portillo CJ, Coggins T, Davis H, Pullinger CR, et al. Types of sleep problems in adults living with HIV/AIDS. J Clin Sleep Med 2012;8:67-75. http://dx.doi.org/10.5664/jcsm.1666

17. Wibbeler T, Reichelt D, Husstedt IW, Evers S. Sleepiness and sleep quality in patients with HIV infection. J Psychosom Res 2012;72:439-42. http://dx.doi.org/10.1016/j.jpsychores.2012.03.003 18. Happe S, Kundmüller L, Reichelt D, Husstedt IW, Evers S. Comorbidity of restless legs syndrome and HIV infection. J Neurol 2007;254:1401-06. http://dx.doi.org/10.1007/s00415-007-0563-2

19. Hennessy MD, Zak RS, Gay CL, Pullinger CR, Lee KA, Aouizerat BE. Polymorphisms of Interleukin-1 Beta and Interleukin-17Alpha Genes Are Associated With Restless Legs Syndrome. Biol Res Nurs 2014;16:143-51. http://dx.doi.org/10.1177/1099800413478827

20.Gamaldo CE, Spira AP, Hock RS, Salas RE, McArthur JC, David PM, et al. Sleep, function and HIV: a multi-method assessment. AIDS Behav 2013;17:2808-15. http://dx.doi.org/10.1007/s10461-0120401-0

21.Vosvick M, Gore-Felton C, Ashton E, Koopman C, Fluery T, Israelski $\mathrm{D}$, et al. Sleep disturbances among HIV-positive adults: the role of pain, stress, and social support. J Psychosom Res 2004;57:459-63. http://dx.doi.org/10.1016/j.jpsychores.2004.03.003 22. Reid S, Dwyer J. Insomnia in HIV infection: A systematic review of prevalence, correlates, and management. Psychosom Med 2005;67:260-9.

http://dx.doi.org/10.1097/01.psy.0000151771.46127.df

23.Seay JS, McIntosh R, Fekete EM, Fletcher MA, Kumar M, Schneiderman $N$, et al. Self-reported sleep disturbance is associated with lower CD4 count and 24-h urinary dopamine levels in ethnic minority women living with HIV. Psychoneuroendocrinology 2013;38:2647-53.

http://dx.doi.org/10.1016/j.psyneuen.2013.06.022

24.Weinstock LB, Walters AS, Paueksakon, P. Restless legs syndrome-theoretical roles of inflammatory and immune mechanisms. Sleep Med Rev 2012;16:341-54. http://dx.doi.org/10.1016/j.smrv.2011.09.003

25.Lee KA, Gay C, Pullinger CR, Hennessy MD, Zak RS, Aouizerat BE. Cytokine Polymorphisms are Associated with Poor Sleep Maintenance in Adults Living with Human Immunodeficiency Virus/Acquired Immunodeficiency Syndrome. Sleep 2014;37:453-63. http://dx.doi.org/10.5665/sleep.3474 